\title{
ARTÍCULO
}

\section{Cátedra Ingenio, Ciencia, Tecnología y Sociedad, una experiencia de ingeniería humanitaria.}

\author{
León, Andrés Leonardo*, Molina-Soler, Angélica ${ }^{\circ}$ \\ * Universidad Nacional de Colombia, Sede Medellín, Facultad de Ciencias Humanas y Sociales, \\ alleon@unal.edu.co \\ - Universidad Nacional de Colombia, Sede Bogotá, Departamento de Ingeniería Civil y Agrícola, \\ almolinas@unal.edu.co
}

La cátedra Ingenio, Ciencia, Tecnología y Sociedad es una materia electiva de la Universidad Nacional de Colombia que se dicta a estudiantes de todas las carreras con el objetivo de brindar herramientas que permitan desarrollar iniciativas de impacto social y plantear alternativas para resolver problemáticas en las que la academia incida de manera pertinente y trabaje en conjunto con las comunidades. La materia surgió como una iniciativa estudiantil del grupo de trabajo Ingenio sin Fronteras, junto con el semillero Ciencia, Tecnología y Sociedad. Nació como un curso alternativo y llegó a convertirse en una asignatura oficial de la universidad desde 2014. Cada semestre, se invitan académicos, expertos y miembros de comunidades a compartir sus conocimientos y experiencias, priorizando el diálogo de saberes. Además, se desarrollan actividades que permitan a los estudiantes trabajar en un proyecto, con miras a resolver una problemática con implicaciones tecnológicas en una comunidad.

\section{Palabras clave:}

Educación, Trabajo comunitario, Impacto social, Ingeniería humanitaria, Educación popular.

\section{ANTECEDENTES}

A mediados del 2010, se creó el grupo Ingenio Sin Fronteras (ISF) conformado por estudiantes de la Universidad Nacional de Colombia interesados en poner el conocimiento ingenieril al servicio de las comunidades vulnerables y cuestionar la relación sociedad-universidad. El surgimiento de la iniciativa fue motivado por la participación de algunos estudiantes en los seminarios internacionales de Ingenieros Sin Fronteras Colombia (ISF-COL) (Cortés Mora, Martínez Castro, 
León Rojas, \& Peña Reyes, 2013) y por la sensibilidad producto del trabajo comunitario y el activismo político de algunos representantes estudiantiles ante diferentes instancias en la Facultad de Ingeniería de la Universidad Nacional de Colombia. Aunque el grupo ISF nació bajo la inspiración y el auspicio de ISF-COL, algunos factores - como la consolidación de una identidad propia, las diferencias metodológicas, el interés por incluir personas de otras áreas del conocimiento y la preocupación por el sesgo de género a partir del nombre Ingenieros - hicieron que tiempo después de su creación el grupo tuviera un carácter independiente del capítulo ISFCOL de Engineers Without Borders International. Sin embargo, hasta la fecha ambos grupos han mantenido una relación de colaboración y apoyo mutuo.

Desde el 2010 y hasta el 2014, ISF planteó y desarrolló diferentes actividades dentro y fuera de la universidad en temáticas relacionadas con el trabajo de la ingeniería con diversos sectores de la sociedad. Como resultado, surgieron cursos de fotografía, seminarios y proyectos de extensión solidaria. El grupo creció con la integración de egresados y profesores; aunque la participación de estudiantes fue determinante para la vida, actividad y desarrollo del grupo.

En el 2011, ISF fue el primer ganador en el área de ingeniería en la "Convocatoria Nacional de Extensión Solidaria UN: Apuesta para construir país”, con el proyecto Trashware (León Rojas, 2013). Este proyecto consistía en recuperar partes de computadores usados o devaluados que aún presentaban buenas condiciones de funcionamiento para ensamblar un equipo funcional bajo un sistema operativo de Licencia Pública General (GPL) y con aplicaciones diversas para ofimática, educación, matemáticas y entretenimiento (León, Martínez, Reina \& Romero, 2012). El propósito era ampliar las condiciones de acceso a las tecnologías de la información y comunicaciones (TIC) en comunidades con pocos recursos económicos, mediante la aplicación de algunos elementos planteados en el enfoque de Tecnologías de la Información y las Comunicaciones para el Desarrollo (ICT4D) (Martínez \& Díaz, 2014). Desde el 2012 y durante dos años, el proyecto se realizó en la Institución Educativa Popular Fe y Esperanza en el barrio El Progreso de Altos de Cazucá, municipio de Soacha, Cundinamarca (León Rojas, 2013).

Además, se logró integrar a otros estudiantes de la Facultad de Ingeniería, quienes recibían clases de docentes de la materia Taller de Proyectos Interdisciplinarios (TPI). Ellos hacían parte del proyecto e integraban trabajos de esa asignatura en el desarrollo de Trashware, por ejemplo, la elaboración de una cartilla para la enseñanza del software libre y el diseño de la infraestructura eléctrica de la sala de computadores, el cual fue utilizado en la adecuación del espacio. El proyecto implicó retos pedagógicos, metodológicos, técnicos, organizativos, de implementación y apropiación de la tecnología, ya que la mayoría de niños y jóvenes de la comunidad tenían nula o muy baja familiaridad con los computadores, lo cual generó la necesidad de mejorar la cualificación de los integrantes de ISF.

Los integrantes de ISF definieron algunos intereses de formación a partir de los retos generados por el proyecto Trashware, especialmente en metodologías de trabajo como Investigación Acción Participativa y en el papel de la academia para la transformación social, de tal forma que no se redujera o desviara hacia el asistencialismo. Así mismo, plantearon otras iniciativas de trabajo con comunidades y conocieron referencias internacionales en temáticas de ingeniería y sociedad. Por lo tanto, durante el 2013 se creó un espacio académico denominado Seminario Ingenio Sin Fronteras, acorde a las necesidades e intereses del grupo, pero abierto a la participación de la comunidad académica en la universidad. Las sesiones se desarrollaron durante dos semestres del 2013 con las temáticas: universidad y sociedad; tecnologías alternativas; experiencias de trabajo 
con comunidades; y desarrollo de la ingeniería en Colombia. El Seminario Ingenio Sin Fronteras fue un espacio informal que, aunque contó con una asistencia de público modesta, básicamente los miembros del grupo y no más de diez personas más, permitió salvaguardar la ausencia de espacios de reflexión, discusión y acción para el trabajo con comunidades vulnerables (Reina, Gaitán, Díaz \& Peña, 2015), gestados desde la ingeniería, con un interés por el empoderamiento tecnológico, la organización comunitaria con apoyo de la academia y los estudios sociales de la ciencia y la tecnología.

A mediados del año 2014, se creó el Grupo de Investigación en Tecnologías e Innovación para el Desarrollo Comunitario-GITIDC, debido a la carencia de un espacio formal en la Universidad Nacional que articulara los trabajos e iniciativas que se venían desarrollando desde la ingeniería con elementos de las ciencias sociales y humanas y que tuviera un reconocimiento institucional ante la academia. Este grupo acogería a los integrantes de ISF y contaría con nuevos miembros para la investigación rigurosa y reflexiva en temas como: Universidad y Educación en Ingeniería; Ingeniería y Sociedad; Innovación y Comunidad; e Ingeniería y Sustentabilidad, temáticas en las cuales los integrantes han desarrollado su praxis académica en tesis de maestría, doctorado y trabajos de extensión.

\section{PROPÓSITOS Y FINALIDADES DEL CURSO}

El curso se desarrolla bajo la premisa de que las áreas de ciencia e ingeniería no tienen fundamentos académicos, teóricos, prácticos y metodológicos suficientes para lograr que proyectos con comunidades vulnerables tengan el impacto deseado (Reina-Rozo \& Peña-Reyes, 2015). En ese sentido, la cátedra busca la generación de capacidades en los estudiantes para el desarrollo de proyectos de ciencia y tecnología con comunidades no científicas ni ingenieriles.

Se busca que los estudiantes generen capacidades a partir de: el conocimiento de herramientas para la interacción con diversas poblaciones; la reflexión crítica de la implementación de tecnológicas; el cuestionamiento de la noción de desarrollo; la interacción en entornos reales con problemáticas sentidas; la ampliación de los horizontes de sentido de su profesión; y el desarrollo de habilidades socio-emocionales, de escritura, lectura, trabajo en equipo.

El curso es electivo y no hace parte de ningún programa curricular ni está ofertado de manera exclusiva a alguna carrera. Además, la asignatura no presenta algún requisito previo. Por tal motivo ha contado con una heterogeneidad de estudiantes de diferentes planes de estudio y de diversos porcentajes de avance, aunque la población universitaria que ha cursado la materia ha sido en su mayoría estudiantes con una relación más directa con las ciencias naturales y la tecnología, éstos atraídos por el nombre de la asignatura. El curso ha buscado que los estudiantes realicen una valoración crítica de lo aprendido durante su tiempo en la universidad y lo pongan al servicio de la construcción de una sociedad más incluyente.

El curso ha propiciado ambientes de aprendizaje mediados por la interdisciplinariedad, a partir de la formación de grupos de aproximadamente cinco estudiantes de diversas disciplinas para la planeación o ejecución de un proyecto durante todo el semestre. Así mismo, el equipo gestor ha tenido formación en diferentes disciplinas de la ingeniería y las ciencias naturales, con un interés por las ciencias sociales y los estudios sociales de la ciencia y la tecnología, lo que permite que los participantes cuenten con dicho asesoramiento y orientación. 
Inspirados por la teoría constructivista y pedagogías centradas en el aprendizaje activo, la cátedra adapta elementos del Aprendizaje Basado en Proyectos (PBL por sus siglas en inglés) al intentar resolver un problema real, en un determinado contexto. Por ejemplo, desde el 2017 en la asignatura ha habido una mayor preocupación por contar con comunidades con algún grado de organización que tengan un problema susceptible de ser solucionado por estudiantes, en un semestre académico. El acercamiento se hace a partir del trabajo previo que han tenido los integrantes del grupo gestor, los colectivos estudiantiles cercanos y quienes inscriben la materia con esas organizaciones sociales. Además, son los estudiantes junto a las comunidades quienes delimitan y definen el proyecto. Por esto el rol de docente se transforma en un facilitador y asesor. El estudiante de la cátedra se expone a ambientes de enseñanza y aprendizaje experienciales y vivenciales.

Bajo el principio formativo de la dialogicidad, la cátedra incorpora elementos de la pedagogía de la liberación, desarrollada por Paulo Freire (Freire, 1979). El diálogo en Freire tiene el potencial de problematizar una situación con un interés de transformación de la realidad. Es por esto que el diálogo en la asignatura es un punto de partida que, aunque ha sido planeado y tiene una intención, es flexible de acuerdo a la dinámica propia en que se desarrolle. Así como se han dado diálogos entre los mismos integrantes del grupo, los estudiantes con los miembros de las comunidades, con los conferencistas invitados y con el equipo gestor. El aprendizaje se ha dado en una doble vía. Por ejemplo, el equipo gestor aprende con y de los estudiantes, tratando de comprender sus intereses, limitaciones, aspiraciones y capacidades, buscando un mejoramiento del curso acorde al contexto y los estudiantes aprenden de sus propios compañeros de clase, de experiencias significativas de otros, de la formulación de políticas públicas, de conocimientos teóricos y perspectivas históricas.

La ingeniería humanitaria ha alimentado e inspirado el curso. Nociones como el co-diseño (Southern et. al, 2014) han tomado una mayor relevancia en el momento de plantear posibles soluciones a las problemáticas que acarrean un tercero. La preocupación de generar proyectos donde primen criterios de solidaridad y justicia social, mas no de caridad, han sido centrales en el curso. Adicionalmente, el equipo gestor de la cátedra ha tenido el interés por que los estudiantes vislumbren otros posibles horizontes profesionales, que trascienden la incorporación en empresas o industrias.

La cátedra no se concibe como un proyecto acabado sino un proceso en continua construcción, elaborado a partir de la interacción de referentes académicos y sociales e incluso del proceso mismo en el aula de clase. Se ha re-alimentado a partir de evaluaciones de los estudiantes, de profesores externos y del mismo grupo gestor, lo cual ha llevado, entre otras cosas, a modificar el ensayo que se pide, a cambiar algunos ponentes, a modificar dinámicas de grupo y a hacer actividades más prácticas. Siempre con el objetivo de acercar los logros de la ciencia y la tecnología a poblaciones excluidas por estos campos de conocimiento y de acercar la sociedad a la academia, además de reflexionar las implicaciones sociales de la generación de artefactos y desarrollos científicos.

\section{RECUENTO HISTÓRICO}

A finales del 2013, la cátedra Ingenio, Ciencia, Tecnología y Sociedad nació como asignatura oficial de libre elección y como una formalización del Seminario Ingenio Sin Fronteras. La cátedra fue posible gracias al aval, el apoyo y el respaldo del profesor y luego decano de la Facultad de Ingeniería José Ismael Peña, quien incitó y motivó la constitución y formalización de la asignatura en la Facultad. Para ese entonces, varios miembros del grupo ISF eran estudiantes de maestría y 
doctorado, interesados en hacer estudios rigurosos sobre la relación de la tecnología y la sociedad. En aquel momento, la cátedra estuvo bajo la coordinación de integrantes del grupo Ingenio Sin Fronteras (ISF) y del grupo Ciencia, Tecnología y Sociedad (CTS), este último perteneciente a la Facultad de Ciencias, que se unió a este propósito. Por esa razón, el nombre de la cátedra fue dado por la conjugación de ambas iniciativas.

Desde el primer semestre del 2014 y hasta la fecha (2018), la cátedra ha sido ofertada ininterrumpidamente durante todos los semestres académicos y cursada por estudiantes de pregrado de varios programas académicos, de todas las facultades de la universidad, aunque principalmente de Ingeniería y Ciencias. En el transcurso de los semestres han habido cambios en las temáticas, la metodología del curso, la forma de evaluación, la manera de acercarse a las comunidades, el eje transversal que enmarca contextualmente la asignatura y los agentes de coordinación y gestión.

En la Universidad Nacional de Colombia, la asignatura ha sido ofertada por la Facultad de Ingeniería - Sede Bogotá. Sin embargo, en el segundo semestre del año 2015 existió en paralelo una versión del mismo curso en la Facultad de Ciencias Humanas y Económicas en la sede Medellín, a cargo de un miembro del grupo ISF (hoy GITIDC). Por otro lado, los anteproyectos y proyectos planteados y desarrollados trascienden una ubicación específica: aunque mayoritariamente han trabajado con población de Bogotá, comunidades en otros territorios colombianos han participado, como en los departamentos de La Guajira, Tolima y Cundinamarca.

Para el primer semestre del 2016, el curso fue ganador de la convocatoria "Cátedras de Sede" por parte de la Dirección Académica de la universidad, bajo la modalidad de Cátedra de Sede José Celestino Mutis; asignatura que nació en la universidad en conmemoración del bicentenario del fallecimiento del naturalista y científico José Celestino Mutis, en el año 2007, con el objetivo de crear un espacio para la reflexión y la discusión sobre la ciencia y la tecnología como factores fundamentales en el desarrollo del país (Acuerdo 17 de 2007 Consejo de sede). Las cátedras de sede buscan la interdisciplinariedad y ampliación de las distintas perspectivas en temas de interés para la comunidad académica. Para la versión 2016-1, el curso tomó el nombre de Cátedra de Sede José Celestino Mutis - Ingenio, Ciencia, Tecnología y Sociedad: diálogos y retos en Colombia.

La conversión de curso a una cátedra de sede permitió ampliar el número de estudiantes que tomaron la materia. Pasó de 60 a 300 inscritos. Además, contó con recursos financieros para la financiación de gastos de viaje y viáticos de profesores nacionales e internacionales, el servicio de una página web (http://www.catedras-bogota.unal.edu.co/index.php/Mutis), el diseño y divulgación de material y el pago a estudiantes auxiliares.

Durante las nueve versiones de la cátedra se ha contado con la participación de docentes, expertos académicos, investigadores, activistas, líderes sociales, representantes de organismos no gubernamentales (ONG) y organizaciones sociales de origen nacional e internacional que de manera voluntaria han compartido sus conocimientos y experiencias con los estudiantes. Solamente en el primer semestre del año 2016 se les brindó un apoyo económico a los diferentes conferencistas. Sin embargo, la imposibilidad de contar con un recurso monetario para los invitados no ha sido impedimento para la participación de un buen número de expositores y panelistas, entre ellos, invitados internacionales de Ecuador, Argentina, Estados Unidos, Reino Unido, Dinamarca y Brasil, con referentes en la cultura libre como Mariano Fressoli de la Universidad de Quilmes o de la ingeniería humanitaria como Juan Lucena de la Escuela de Minas de Colorado. Esta cátedra es evidencia empírica de la solidaridad y apoyo mutuo entre seres humanos y sus redes. Los 
invitados encuentran un lugar en la academia para expresar sus ideas y los estudiantes se enriquecen con la ampliación y contextualización de la realidad.

La cátedra Ingenio, Ciencia, Tecnología y Sociedad ha ido cambiando su eje transversal. Por ejemplo, en el 2016-2 se enfocó en la región del Sumapaz donde la universidad está desarrollando un programa de admisión especial para habitantes del área rural de Bogotá y en el 2017-2 se trabajó con especial interés el tema del Acuerdo de Paz. El contenido del curso siempre ha estado enmarcado por la relación de la triada: sociedad, tecnología y ciencia, pero ha cambiado algunas temáticas específicas. Se puede resaltar que desde el segundo semestre del año 2015 se incluyó una sesión en estudios de género y feminismo relacionados con ciencia y tecnología.

Desde su ideación hasta la fecha, la cátedra ha sido un esfuerzo de gestión grupal. Durante estos años, ISF (hoy GITIDC) ha formado un grupo de interesados en el curso que, bajo la coordinación de un "responsable", ha trazado y desarrollado el curso. Es así como en un inicio contó con miembros del grupo CTS, quienes acompañaron el curso un par de semestres. Desde el 2017 se cuenta con el respaldo y apoyo de integrantes del Centro de Alternativas al Desarrollo (CEALDES) y durante toda su historia ha contado con la participación de estudiantes de pregrado, maestría, doctorado y profesores de planta en este grupo de coordinación y gestión, quienes se han involucrado de diferentes maneras al apoyo del curso. Adicionalmente, la responsabilidad institucional no ha sido estática, de manera que, hasta el primer semestre de 2018, cinco personas han asumido formalmente como docentes de la cátedra: José Ismael Peña Reyes, Juan David Reina Rozo, Julio César Cañón, Angélica Molina Soler y Andrés Leonardo León Rojas.

En los primeros semestres, en el curso se trabajaron anteproyectos como una forma de aproximación teórica al trabajo con comunidades por dos razones fundamentales: no había experiencia suficiente en el grupo gestor para hacerlo sin mayores errores; y no se quería que las comunidades fueran o se sintieran manipuladas por la academia de forma extensionista, pues esta es una acción antidialógica (Freire, 1973) en donde el conocimiento fluye en una sola vía. Luego de la experiencia de trabajo de algunos años en la cátedra y en proyectos de este tipo, se resolvió que a partir de 2017-2 se trabajaría con comunidades a partir de un banco de problemáticas que se elaborara desde o con ellas, de modo que se diera un diálogo horizontal en donde primara la confianza y la humildad (Freire, 1971) en búsqueda conjunta de soluciones a problemas reales, entendiendo que el conocimiento se construye mediante las relaciones del ser humano en el mundo, con miras a su transformación, y además se perfecciona por medio de la problematización crítica de estas relaciones (Freire, 1973).

\section{HERRAMIENTAS METODOLÓGICAS}

La cátedra ha implementado diferentes técnicas participativas y herramientas metodológicas, las cuales se han adaptado y transformado durante el tiempo de existencia. Algunas de éstas son:

Charlas magistrales, foros y paneles: a cargo de invitados de la academia y de procesos sociales que trabajan en los temas contemplados, que incentiven la discusión y práctica de los anteproyecto y proyectos desarrollados.

Ensayos: en la cátedra los estudiantes de manera individual han desarrollado escritos en prosa para analizar, interpretar o evaluar temáticas desarrolladas en el curso. Por medio del ensayo integran reflexiones planteadas en clase e integran lecturas sugeridas. 
Teatro del oprimido: orientado por un estudiante de ciencias humanas, quien había sido monitor de la cátedra. Se realiza un taller práctico del teatro del oprimido bajo la filosofía de Augusto Boal (Boal, 2011). Este autor plantea que por medio del lenguaje corporal se generan otras formas de comunicación con el otro y con el entorno de modo que, sin importar el grado de escolaridad, las personas puedan expresarse de forma más libre, entender relaciones de poder en su cotidianidad en búsqueda de su transformación. La clase comienza con ejercicios individuales, luego se va entrando en diálogo no verbal con los demás, hasta construir un escenario performativo en donde se pongan en cuestión situaciones de injusticia que han sido normalizadas por la cultura.

Juego de roles: para la construcción del anteproyecto, en los grupos de trabajo se genera un escenario en el cual los miembros interpretan a cada uno de las partes más interesadas en que el proyecto pueda o no ser llevado a cabo de forma exitosa. De esa manera se trata de comprender los intereses particulares de cada sector, entendiendo que un problema nunca se relaciona exclusivamente con aspectos técnicos sino que hay relaciones de poder que facilitan, obstruyen u orientan un plan determinado, por lo que es necesario desarrollar capacidades de negociación y traducción para cada sector involucrado.

Camino del privilegio: mediante una actividad lúdica se pretende mostrar que cada persona tiene un punto de partida distinto en la sociedad, que enfrenta distintas ventajas o desventajas frente a los demás. La actividad complejiza la responsabilidad individual como único factor determinante para el "éxito" social y la desigualdad de la competencia en la sociedad capitalista. El ejercicio pretende reflexionar y derrumbar los mitos de la meritocracia y el de "el que es pobre es porque quiere".

Cartografía social: es un ejercicio para que los estudiantes construyan la representación de un lugar con elementos geográficos, sociales y emocionales. El mapa busca materializar la construcción social de un entorno por parte de sus involucrados e intereses. Los estudiantes dibujan su entorno, en este caso la universidad, identificando lugares de acuerdo a prioridades ambientales, políticas, culturales y situaciones que se presenten allí que puedan ser mejoradas.

Visita al Centro de Innovación de Tecnologías Apropiadas y Educación C-Innova, afiliado a la Red de Innovación para el Desarrollo Internacional IDIN: los estudiantes cambian el aula de clase para participar de charlas o talleres en el centro ubicado en cercanía al campus universitario. El centro ha permitido reflexionar desde una mirada novedosa sobre el quehacer de las disciplinas en la solución de problemas sociales del entorno. Por ejemplo, se han implementado proyectos realizados durante la visita donde los estudiantes han intervenido calles (Molina-Soler, León \& Peña, 2017), elaborado lámparas de bajo costo o aprendido acerca del Desarrollo de la Capacidad Creativa (Creative Capacity Building).

\section{APRENDIZAJES}

La primera enseñanza que se consiguió en la preparación del curso es que sí es posible construir academia alternativa desde adentro. Lo que surgió como una iniciativa estudiantil se logró consolidar, formalizar y proyectar, demostrando que la ingeniería en el contexto colombiano dominada por la ideología neoliberal, diseñada para satisfacer los intereses del mercado, tiene 
Cátedra Ingenio, Ciencia, Tecnología y Sociedad, una experiencia de ingeniería humanitaria.

fisuras que permiten construir alternativas que respondan a las necesidades de las amplias capas de la sociedad, desde la misma institucionalidad.

La cátedra se construyó de forma que haya un aprendizaje permanente no solo para los estudiantes sino para el grupo gestor, haciendo que los contenidos de un semestre varíen significativamente, lo que permite un proceso continuo de diálogo con nuevos saberes, de forma que no se vea la materia como algo inalterable en el tiempo. En ese sentido, se cambian ciertas temáticas según los intereses y necesidades del grupo gestor, se han ido integrando nuevos actores y se ha evidenciado que cuando hay convivencia con la comunidad, los proyectos salen de mejor manera pues se elevan los niveles de compromiso de los estudiantes.

Al poner el diálogo de saberes en primer orden, los aprendizajes se dan en varias vías, no solo del profesor al estudiante, sino que se construye una relación dialéctica en la que el conocimiento fluye desde y hacia todas las partes, incluyendo a las comunidades que se hacen presentes tanto en las clases como en el desarrollo de los proyectos. Ejemplo de ello ha sido el proyecto de emisoras comunitarias en la vereda Puerto Matilde, en el Magdalena Medio, el cual fue diseñado en conjunto entre el grupo GITIDC y la Asociación Campesina del Valle del Río Cimitarra a partir de las necesidades y experiencias de la población, así como de los conocimientos e intereses del grupo. En las distintas fases ha habido un diálogo y una construcción permanente entre todos, con visitas a la región, talleres en doble vía, logrando estrechar lazos y aprender tanto del saber académico como del popular.

\section{RETOS Y DESAFÍOS}

El principal desafío en cuanto a los proyectos es lograr que éstos no queden limitados al espacio de un salón y al tiempo de un semestre académico sino que puedan trascender más allá, permitiendo que puedan continuar en otras versiones del curso, en otras materias, que se puedan proyectar como prácticas profesionales o trabajos de grado, llegando incluso a ver la manera de que se construyan proyectos de vida poniendo a la ciencia y la tecnología al servicio de las comunidades y no solo a la industria. En ese sentido, se proyecta la creación de un semillero de investigación para que quienes han pasado por la cátedra puedan continuar desde la academia en los proyectos y construir unos nuevos con las comunidades.

Se tendría como ideal el hecho de que se integren este tipo de materias interdisciplinarias entre ciencia, ingeniería y humanidades a los currículos de las carreras tanto en pregrado como en maestría, pudiendo de esta forma abrir no sólo las ciencias sociales (Wallerstein, 1996) sino el saber académico en su conjunto, superando las visiones fragmentadas y sectarias que rigen la universidad como atavismo medieval, evidenciado en la extrema burocracia que entorpece los cambios pero que a la vez puede llegar a fortalecerlos.

\section{TRABAJO FUTURO}

A partir de la experiencia de la cátedra como electiva, tanto en Bogotá como Medellín, así como cuando fue cátedra de sede José Celestino Mutis, desde la organización del curso se planteó la transformación completa, creando desde ahí dos asignaturas de libre elección con la posibilidad de que un grupo importante de estudiantes puedan inscribir ambas. Una estaría orientada a la parte teórica, donde se discuta el papel de la ciencia y la tecnología en la transformación de la realidad 
Cátedra Ingenio, Ciencia, Tecnología y Sociedad, una experiencia de ingeniería humanitaria.

bajo el principio de la justicia social. La otra sería más práctica y se desarrollarían proyectos concretos en comunidades cercanas o pertenecientes a Bogotá con miras a resolver un problema concreto en el que esté presente la ciencia y la tecnología como elementos centrales, sin obviar las herramientas metodológicas que puedan ser necesarias para conseguir ese objetivo.

\section{REFERENCIAS}

Boal, A. (2011). Teatro oprimido. Cuadernos de Pedagogía, (411), 46-51.

Cortés Mora, H. G., Martínez Castro, D. F., León Rojas, L. R., \& Peña Reyes, J. I. (2013). Ingenio Sin Fronteras. Una alternativa de extensión solidaria de la Universidad Nacional de Colombia. Paper presented at the Eleventh LACCEI Latin American and Caribbean Conference for Engineering and Technology (LACCEI'2013) International Competition of Student Posters and Paper, Cancun, Mexico.

Freire, P. (1979). La Pedagogía del Oprimido. México: Siglo Veintiuno.

Giraldo, O. F. (2008). Seguridad alimentaria y producción pecuaria campesina: el caso de la localidad rural de Sumapaz. Revista Luna Azul, (27).

Hermes, (s.f.) Sistema de Información de la Investigación Universidad Nacional de Colombia. Disponible en: http://www.hermes.unal.edu.co/pages/Consultas/Grupo.xhtml?idGrupo=2224\&opcion=1

Pérez Martínez, M. E. (2008). La adaptabilidad de pobladores y asentamientos rurales en áreas de conurbación: El caso de la ciudad de Bogotá (Colombia). Cuadernos de desarrollo rural, 5(60).

León, A. L., Martínez, D. F., Reina, J. D., \& Romero, J. A. (2012). Trashware para el desarrollo social y sostenible. In Congreso Colombiano de Cultura Libre (pp. 1-9).

León Rojas, A. L. (2013). Caracterización de la extensión solidaria en la Universidad Nacional de Colombia. (Magíster), Universidad Nacional de Colombia, Bogotá.

Martínez, D., \& Díaz, H. (2014). Proyecto Trashware: Teorías, Aplicación, Evaluación y lecciones aprendidas. In Workshop: South-North-South dialogues on Science, Technology and Development (pp. 1-14).

Molina-Soler, A., León, A. L. \& Peña-Reyes, I (2017). Ingeniería humanitaria en el aula: aprendizaje desde la sistematización de experiencias en el Centro de tecnologías apropiadas y educación," presentado en el Coloquio internacional interdisciplinario Colombia - Francia. La Ciudad-Región sostenible como proyecto: desafíos actuales. Visiones cruzadas y perspectivas., Bogotá, Colombia.

Reina-Rozo, J. D.; Peña-Reyes, Ismael. (2015). Inventiveness and Society, an experience from the Problem Based-Learning approach for a post-conflict scenario in Colombia. En: De Graff, Erik et al. (eds.). Global Research Community: Collaboration and Developments. Aalborg: Aalborg University Press, p. 245-256.

Reina, J. D., Gaitán Albarracín, N., Díaz Avendaño, H. A., \& Peña Reyes, J. I. (2015). Ingenio y Sociedad: Hacia una educación de Ingeniería Humanitaria en Colombia. Encuentro Internacional de Educación en Ingeniería, Cartagena (Colombia). 
Cátedra Ingenio, Ciencia, Tecnología y Sociedad, una experiencia de ingeniería humanitaria.

Southern, J., Ellis, R., Ferrario, M. A., McNally, R., Dillon, R., Simm, W., \& Whittle, J. (2014). Imaginative labour and relationships of care: Co-designing prototypes with vulnerable communities. Technological Forecasting and Social Change, 84, 131-142.

Wallerstein, I. (Ed.). (1996). Abrir las ciencias sociales: informe de la Comisión Gulbenkian para la reestructuración de las ciencias sociales. Siglo XXI. 